DOI: https://doi.org/10.34069/AI/2021.45.09.8

How to Cite:

Goicochea Morales, L.B., \& Kapustina, D.M. (2021). Particularidades de las réplicas en el proceso de la comunicación lingüística intercultural en la etapa inicial de la conversación telefónica, entre representantes de los países hispano y ruso-hablantes. Amazonia Investiga, 10(45), 82-87. https://doi.org/10.34069/AI/2021.45.09.8

\title{
Particularidades de las réplicas en el proceso de la comunicación lingüística intercultural en la etapa inicial de la conversación telefónica, entre representantes de los países hispano y ruso-hablantes
}

\section{Особенности ответов в процессе межкультурного лингвистического общения на начальном этапе телефонного разговора между представителями испаноязычных и русскоязычных стран}

Received: July 27, 2021
Accepted: September 12, 2021

Written by:

Lorenzo Benjamín Goicochea Morales ${ }^{29}$

https://www.elibrary.ru/author_profile.asp?id=613601 https://orcid.org/0000-0002-2840-685X

Daria M. Kapustina ${ }^{30}$

https://www.scopus.com/authid/detail.uri?authorId=57224223077 https://www.elibrary.ru/author_profile.asp?id=593275 https://orcid.org/0000-0001-7236-8937

\section{Resumen}

En el presente trabajo se lleva a cabo un análisis de la literatura sobre el problema de la comunicación intercultural, incluyendo la conversación telefónica, entre representantes de los países hispano y ruso-hablantes. Cobra especial relevancia el análisis de las peculiaridades de las réplicas en el proceso de la comunicación lingüística intercultural en la etapa inicial de una conversación telefónica, entre representantes de países hispanohablantes y de habla rusa, ya que contribuye a la interacción de las culturas y al enriquecimiento del acervo lingüístico de sus participantes. El objetivo del presente trabajo fue analizar las réplicas clave durante las conversaciones telefónicas entre representantes de varios países de habla hispana. El autor utiliza réplicas de clichés en diferentes etapas de una conversación telefónica y describe las razones para elegir una u otra réplica. Además, el autor presta especial atención a las réplicas usadas en el inicio de la conversación telefónica. El principal método de investigación para obtener información sobre el tema del trabajo fue la entrevista realizada con interlocutores hispanos sobre temas específicos. Además, se realizó un análisis de contenido de las conversaciones telefónicas para identificar las réplicas más repetitivas.

\begin{abstract}
Аннотация
В данной работе проводится обзор литературы по проблеме межкультурной коммуникации в том числе во время телефонного разговора между представителями испаноязычных и русскоязычных стран. Анализ особенности реплик в процессе лингвистической межкультурной коммуникации на начальном этапе телефонного разговора между представителями испаноязычных и русскоязычных стран становится особенно актуальным, так как способствует взаимодействию культур и обогащению лингвистического запаса у субъектов общения. Цель работы состояла в анализе ключевых реплик при телефонных разговорах с представителями различных испаноязычных стран. Автором проводится использование реплик-клише на разных этапах телефонного разговора и описываются причины выбора той или иной реплики. Ocобое внимание автор уделяет репликам, касающихся начала разговора. Основным методом исследования для получения информации по теме работы было интервьюирование, проводимое с испаноязычными собеседниками на заданные темы. Далее был проведен контент-анализ
\end{abstract}

\footnotetext{
${ }^{29}$ Senior Lecturer, Moscow Aviation Institute (National Research University), Moscow, Russia.

${ }^{30} \mathrm{PhD}$ in Philosophy, Associate Professor, Moscow Aviation Institute (National Research University), Moscow, Russia.
} 


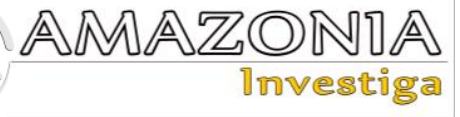

Palabras clave: comunicación intercultural, comunicación lingüística, lengua y cultura, conversación telefónica, réplicas, comportamiento del habla de una persona, diálogo.

\section{Introducción}

Una de las disciplinas más dinámicamente demandadas por la sociedad en el mundo moderno es la comunicación intercultural, que, junto con la sociolingüística, estudia la interacción entre lengua y cultura (Kurbakova et al., 2020). En las investigaciones modernas se enfatiza el hecho que en el transcurso de los últimos años, el desarrollo de la comunicación intercultural ha llegado a ser la dirección principal en la enseñanza de idiomas extranjeros (Yi, 2020).

La comunicación intercultural es estudiada a nivel interdisciplinario, por tales ramas del conocimiento como filosofía, culturología, psicología, lingüística, etnología, antropología, sociología, ciencias políticas, ecología de los medios de comunicación, etc. (Sinyachkin, 2017). El renovado interés en estas disciplinas se debe a la situación geopolítica del mundo (Prikhozhaya, 2019).

La comunicación se ha convertido en objeto de estudio independiente de las ciencias sociales gracias al desarrollo de medios técnicos de transmisión de información -principalmente la radio, desde comienzos de los años 20 del siglo XX. El desarrollo de la teoría de la comunicación fue facilitado por la formación de la cibernética, la informática y la semiótica; ciencias que con el término "comunicación" designan determinados canales técnicos y medios de comunicación. La comunicación se analiza como un sistema multicomponente, compuesto por elementos. El conocimiento comunicativo es interdisciplinario. Por ello, la comunicación es objeto de disciplinas socio-humanitarias, naturales-científicas y científico-técnicas; cada una de las cuales elige de la comunicación su objeto y tema de investigación. Las disciplinas técnicas estudian las posibilidades y métodos de transferir, procesar y almacenar información. Las ciencias sociales (psicología, lingüística, psicolingüística, sociología, sociolingüística, teoría de la comunicación, ciencias políticas, estudios culturales, etnografía) investigan sus aspectos de la comunicación con sus características inherentes y específicas. Así, la lingüística se телефонных разговоров с целью выявления наиболее повторяющихся реплик.

Ключевые слова: межкультурная
коммуникация, лингвистическая
коммуникация, язык и культура, телефонный
разговор, реплики, речевое поведение
личности, диалогическая речь.

ocupa de los problemas de la comunicación verbal, la psicología y la psicolingüística estudian las razones, las barreras que impiden el proceso de comunicación, así como la motivación del comportamiento verbal y no verbal de los comunicantes (Artyushina et al., 2021).

El objetivo del presente trabajo fue analizar las réplicas clave durante las conversaciones telefónicas entre representantes de varios países de habla hispana. El autor utiliza réplicas de clichés en diferentes etapas de una conversación telefónica y describe las razones para elegir una u otra réplica. Además, el autor presta especial atención a las réplicas usadas en el inicio de la conversación telefónica.

\section{Revisión de literatura}

En la actualidad, el aprendizaje de idiomas como medio para aumentar la conciencia intercultural y la comunicación, así como para promocionar el crecimiento profesional de los ciudadanos es uno de los principales objetivos de los sistemas educativos en todo el mundo (Palacios-Hidalgo et al, 2021).

Por esta razón, la tarea principal de la educación moderna es desarrollar las habilidades de los estudiantes para interactuar con otras culturas con éxito, así como responder eficazmente a las demandas, desafíos y oportunidades que surgen en situaciones interculturales (Tsymbalyuk et al., 2021).

Hoy en día, un gran número de trabajos está dedicado a los problemas de la comunicación intercultural (Ponyaeva, 2021; Nazartseva et al., 2019). La doctora Tinyakova E.A. señala que la comunicación lingüística generada por la actividad humana, puede convertirse en un factor capaz de controlarla. La diversidad y complejidad de la comunicación lingüística dificulta aún más los problemas sociales y culturales. Analizando los problemas de la comunicación intercultural, Schoenberger I.I. describe las razones de la ocurrencia de 
malentendidos a nivel lingüístico en el proceso de la comunicación intercultural (Shenberger, 2008). Vladimirova T.E. estudia la comunicación intercultural en un mundo globalizado, a través del prisma de la lingüística. En sus investigaciones, se observa un intento de analizar los conceptos de valor y el comportamiento del habla de una personalidad lingüística nacional como sujeto de comunicación y concluye dando importancia a la formación de una personalidad lingüística bilingüe durante la enseñanza de una lengua extranjera (Vladimirova, 2012). Abdukadyrova T.T. y Tsutsashvili T.A. ofrecen una comprensión científica de los problemas metodológicos y teóricos del aspecto lingüístico en la comunicación intercultural. Sus trabajos se centran en el estudio de los aspectos lingüísticos que pueden influir en la comunicación entre hablantes de diferentes idiomas (Abdukadyrova and Czuczashvili, 2020). El aspecto lingüístico no se limita a identificar las particularidades semánticas de las palabras en diferentes idiomas. También abarca la comparación de diversas situaciones comunicativas, las formas de dividir el mundo por medios lingüísticos, la comparación del comportamiento del habla de representantes de diferentes culturas (Abakumova, 2013). Teniendo en cuenta el componente sociocultural, la comparación de lenguas rusa y extranjeras, revela la profundidad de las diferencias entre lo que hay detrás de las palabras de estas lenguas, es decir, entre las ideas culturales sobre objetos reales y fenómenos de la realidad y entre los objetos y fenómenos en sí mismos (Vedenskaya, 2015).

En los trabajos de T.B. Radbil, se estudia el problema del análisis lingüístico de la especificidad nacional-cultural de las estrategias del habla-comportamiento, cuya expresión lingüística son las peculiaridades de la entonación y la prosodia frasal, las formas idiomáticas de expresar diferentes fuerzas ilocucionarias (intenciones comunicativas ) del hablante, así como los diferentes tipos de "palabras del discurso" (palabras introductorias y modales, partículas, interjecciones) y unidades de metatexto (Radbil, 2014). El diálogo intercultural como el componente más importante de la sociedad moderna, debe contribuir a la autoidentificación de la persona en el espacio cultural (Seregina et al., 2019).

En el centro de investigación de la lingüística moderna está el lenguaje en acción, en primer lugar, la consideración de los patrones de comportamiento del habla de una persona, que está estrechamente relacionado con el problema de la cultura del habla. Hablar por teléfono ocupa un lugar importante en la comunicación moderna (Krasilnikova, 2014).

La conversación telefónica es una de las variedades del habla dialógica, durante la cual tiene lugar una conversación bidireccional y no visual. En la lingüística moderna, se viene realizando una investigación intensiva sobre varios parámetros de una conversación telefónica, se lleva a cabo un análisis de los medios lingüísticos utilizados durante su realización. El enfoque que hacen los científicos del material (conversaciones telefónicas) como de un discurso oral, es legítimo y apropiado. Permite analizar los rasgos fono-estilísticos del habla asociados a la posible influencia de factores extralinguíísticos, para desarrollar un modelo tipológico de conversación telefónica, teniendo en cuenta las condiciones de comunicación y las características de los hablantes (Abakumova, 2013).

Se considera que la primera llamada telefónica fue realizada el 10 de marzo de 1876 por Alexander Graham Bell. De hecho, el teléfono fue inventado por el italiano Antonio Meucci, quien solicitó una patente para su invento allá por 1871, 5 años antes de que lo hiciera el científico escocés. La fama de A. Bell se explica por el hecho de que fueron él y la Bell Company que el mismo fundó, quienes contribuyeron a la popularización de la telefonía y al desarrollo generalizado de la industria telefónica. Bell hizo una demostración de transmisión eléctrica del habla al hacer una llamada a su asistente eléctrico, Thomas Watson. Se produjo entonces la primera conversación entre dos personas que se encontraban en salas distintas y fue recordada para siempre lo dicho: “¡Watson, ven! Te necesito aquí".

En Rusia, la primera conversación telefónica tuvo lugar en noviembre de 1879 entre las ciudades de San Petersburgo y Malaya Vishero. En nuestros tiempos, este tipo de comunicación se utiliza en todo el mundo cada minuto y, gracias a las tecnologías modernas, puede conectar a personas que se encuentran en los rincones más remotos entre sí. También vale la pena señalar que hoy en día este tipo de comunicación se ha desarrollado aún más, gracias a las tecnologías modernas del Internet, ya que "el Internet y sus servicios se han convertido en una herramienta popular" (Krivova et al., 2021).

Cada uno de los participantes de la comunicación telefónica juega un cierto papel comunicativo: el que llama (denotado en otra terminología como "comunicador, emisor") y la persona requerida 


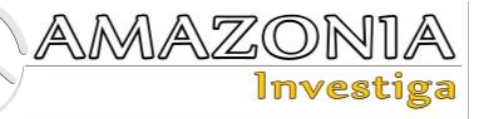

(destinatario, o receptor, que recibe el mensaje del que llama). También se permite la participación de un tercero en una conversación telefónica, que es denominado conectador o mediador. Su función es conectar al comunicador con el destinatario. En este tipo de comunicación se observa claramente que existe una determinada organización de la orientación comunicativa, y al igual que ocurre en la comunicación normal, es importante tener en cuenta el registro estilístico de la conversación. Puede ser oficial, es decir, de trabajo y no oficial, implícito en la comunicación diaria. A. Lindström señala que el comienzo de las conversaciones telefónicas casi siempre es estándar y depende ligeramente de las especificaciones nacionales. Siempre contiene una llamada - una respuesta, una frasereconocimiento, un saludo y una pregunta sobre la salud o los negocios. Las diferencias en la etapa inicial de una conversación telefónica están relacionadas principalmente con el reconocimiento del interlocutor. Además, como bien lo señala T. Pavlidu, es al comienzo de una conversación cuando suelen darse las explicaciones del por qué no respondieron de inmediato, se disculpan por molestar y hablan de una mala audibilidad (Abakumova, 2013).

Gracias a que el comportamiento del habla está determinado por las normas que observan los hablantes, es bastante fácil determinar si una conversación telefónica es formal o informal.

Entre las características inherentes al habla telefónica, podemos destacar las siguientes:

1. Falta de contacto visual entre interlocutores, su ubicación remota;

2. Limitación en el tiempo;

3. Probabilidad de interferencia técnica (mala audibilidad);

4. Los interlocutores se ven privados de la posibilidad de recibir y transmitir comunicaciones no verbales (en particular, elementos del habla cinética como los gestos y las expresiones faciales).

Es indudable que los factores enumerados líneas arriba tienen un impacto directo en la implementación de herramientas lingüísticas y en la realización de los textos de los diálogos telefónicos. El principio comunicativo de la comunicación telefónica se centra en el estándar y la compresión. Son características del habla telefónica varias réplicas estereotipadas (clichés, modelos) (Formanovskaya, 2002).

\section{Métodos}

El principal método de investigación para obtener información sobre el tema del trabajo fue la entrevista realizada con interlocutores hispanos sobre temas específicos. Además, se realizó un análisis de contenido de las conversaciones telefónicas para identificar las réplicas más repetitivas. Se utilizó un conteo segmentario y temático.

El tipo de etnometodología estudiado parte del supuesto de que las conversaciones constituyen la base de otras formas de relaciones interpersonales. Esta es la forma más común de interacción, y cualquier conversación consiste en una matriz coherente de prácticas $y$ procedimientos de comunicación socialmente organizados.

El muestreo consistió en 40 conversaciones en español de contactos personales de los autores con informantes en países como Perú, Argentina, Ecuador, Nicaragua, Cuba y España.

\section{Resultados y discusión}

El análisis llevado a cabo de los estereotipos de réplicas mostró que entre ellos hay no pocos telefónico-especiales. Estos incluyen, por ejemplo, réplicas utilizadas en los países citados, como:

- ¡Aló!

- ¡Comuníqueme con ...!

- ¿Quién habla?

- ¡Bueno!

- ¡Diga!

- ¡No cuelgue!

- ¡Bueno!

- ¿De parte de quién?

Muy a menudo réplicas con incompletitud sintáctico-morfológica de expresión, en las que se omiten elementos semánticamente redundantes:

- ¿¿Con quién?' (en lugar de ‘Con quién desea usted hablar?');

- 'Banco de Crédito del Perú' (en lugar de 'Contesta la secretaría del Banco de Crédito del Perú');

- ¿¿Quién?’ (en lugar de ‘Quién habla?’)

Tales comentarios "fragmentarios" son especialmente abundantes en el registro informal de comunicación. Los comentarios a menudo utilizados, que confirman la aceptación de la información por parte del interlocutor, así como 
el fortalecimiento del contacto verbal en idioma español, son los siguientes:

- $\quad$ Ya, ya (Perú, España);

- ¡Vale! (España);

- Bueno, ¿qué más? (Ecuador);

- ¡No me digas! (Colombia);

- ¡Mira vos! (Nicaragua);

- ¡Ah, ya! (Perú);

- ¡Sí, sí! (Cuba);

- ¿Qué onda, amigo!, ¿Cómo andás? (Argentina).

En la comunicación diaria, estos comentarios suelen ir acompañados de un movimiento de cabeza o expresiones faciales. Al final de una conversación telefónica entre mujeres, a menudo, como etiqueta del habla (EH), se utilizan las siguientes réplicas: ¡Besos! ¡Besitos! ¡Abrazos!

En caso de mala audibilidad, se utilizan tales réplicas repetitivas como:

- $\quad$ ¡Sí, soy yo, Betty! (Perú);

- ¡Hola! ... ¡Hola! (Argentina).

En consecuencia, para el grupo temático de unidades de EH "hablando por teléfono", es típico utilizar no solo unidades de $\mathrm{EH}$ que atienden situaciones de EH más específicas (por ejemplo, trato o dirección, saludo, despedida, disculpa, gratitud), sino también unidades específicas inherentes a esta particular situación comunicativa. En el proceso de las conversaciones telefónicas, es necesario tener en cuenta algunos puntos esenciales que nos ayudarán a describirlos de manera más adecuada.

Refiriéndose a esta temática, el Doctor en Filología VE Gol'din señala que: "una característica de las conversaciones telefónicas es el marco comunicativo obligatorio, cuya parte inicial atiende la llamada del abonado, la identificación de quién está hablando con quién, la aclaración de si existe la posibilidad de entrar en comunicación telefónica en ese momento, la verificación del nivel de audibilidad y algo más" (Gol`din, 2009). El llamado "marco comunicativo" de una conversación telefónica se puede representar mediante un esquema general aproximado, que consta de los siguientes períodos:

1. Establecimiento del contacto inicial de la comunicación;

2. Declaración del propósito de la llamada telefónica. A veces, los interlocutores discuten la información proporcionada;

3. La etapa final de la comunicación telefónica.
El bloque de contenido-información de la comunicación suele incluirse en el segundo período de una conversación telefónica. El diálogo de este período está mucho más liberado de las fórmulas y expresiones estereotipadas que son inherentes a las etapas iniciales y final de la comunicación telefónica.

La variabilidad y el uso de claves-cliché también se utilizan en las conversaciones telefónicas. La elección de una u otra réplica entre las posibles, depende de muchas razones. En el sentido amplio, es la naturaleza estilística de la comunicación -es decir, puede ser formal o informal. Pero existe una serie de factores específicos, entre los que destacamos:

1. Adónde llaman (a casa o a alguna institución);

2. A quién llaman (situación y situación demográfica del destinatario);

3. Quién llama (situación y situación demográfica del emisor);

4. La naturaleza de la relación entre los interlocutores, su estado emocional, su grado de educación y tipo de personalidad mental);

5. En qué país de habla hispana tiene lugar la conversación telefónica (importante para la selección de una serie de unidades lingüísticas).

La réplica característica de la etapa inicial de una conversación telefónica en respuesta a una llamada: la señal inicial para la comunicación, la primera réplica telefónica específica del lado de la persona que ha contestado a una llamada telefónica en los países de habla hispana puede ser una de las siguientes:

- ¡Aló! - Алло!

- ¡Diga! - Говорите!

- ¡Sí! - Да!

- ¡Oigo! - Слышу!

- ¡Hable! (¡Habla!) - Говорите! (Говори!)

- ¡Hola!, (¡Holá!) - Привет!

- ¡Aquí! - Я тут!

- ¡Ajá! - Ага!

- ¡A ver! - Посмотрим!

- ¿Qué hay? - Что нового?

- ¡Bueno! -Хорошо!

- ¿Quién es? - Кто это?

- ¿Quién habla? -Кто это говорит?

- ¡Ordene! - Прикажите!

\section{Conclusión}

Hoy en día se da una importancia creciente a los problemas de la comunicación intercultural, en 


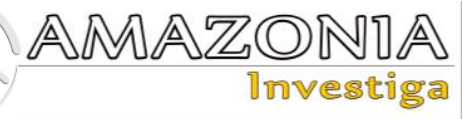

diversos campos del conocimiento. La interacción intercultural entre diferentes países y naciones se está convirtiendo cada vez más en objeto de una atención especial por parte de investigadores y políticos. A lo largo de varios siglos, el diálogo intercultural ruso-español ha ido enriqueciendo el contexto lingüístico de las naciones, haciendo posible comprender mejor la estructura arquitectónica de la cultura y sus cambios profundos. En el mundo moderno, uno de los medios de comunicación intercultural más utilizados es la conversación telefónica. La presente investigación ha permitido identificar las principales réplicas-estereotipos que son usadas en el inicio de las conversaciones telefónicas de habla hispana.

\section{Referencias Bibliográficas}

Abakumova, S. S. (2013). Characteristics of a business conversation on the phone (based on the material of Russian and German languages). Bulletin of the Volgograd State University. Series 2: Linguistics, 1(17), 137-141.

Abdukadyrova, T. T., and Czuczashvili, T.A. (2020). The role of the linguistic aspect in the implementation of intercultural communication. In Proceedings of the XI International Scientific and Practical Conference, 9-14.

Artyushina, G. G., Zhurbenko, N. L., and Sheypak, O. A. (2021). Individual educational path based on learning styles theory as a way to reach sustainable quality education goals. Revista Inclusiones, 8(1), 54-66.

Formanovskaya, N. I. (2002). Verbal communication: a communicative-pragmatic approach. Moscow: Russian language.

Gol'din, V. E. (2009). Conversion: theoretical problems. Saratov: Book House "Librokom".

Krasilnikova, L. V. (2014). Genre varieties of modern telephone conversation. Bulletin of the Center for International Education of Moscow State University. Philology. Culturology. Pedagogy. Methodology, 3, 40-44.

Krivova, A. L., Kalliopin, A. K., Korotaeva, I. E., Shafazhinskaya, N. E., and Ermilova, D. Y. (2021). Social Networks as a Means of Monitoring Students' Progress. Propósitos y Representaciones, 9(SPE3), 1264.

Kurbakova, S. N., Starodubova, O. Y., Khristoforova, N. I., Sabekia, R. B., and Askarova, G. B. (2020). European antiquity in verbal expression: philosophical and cultural aspect. Revista Inclusiones, 7(S1), 337-349.

Nazartseva, E. A., Morya, L. A., and Zubanova, S. G. (2019). Teaching foreign students the vocation-related language. Amazonia Investiga, 8(22), 338-343.

Palacios-Hidalgo, F. J., Huertas-Abril, C. A., and Gómez-Parra, M. E. (2021). Foreign and Bilingual Language Education in the UK and Spain: A Study of Similarities and Differences. Journal of Language and Education, 7(2 (26)), 243-255.

Ponyaeva, T. A. (2021). Innovations in pedagogical activity of higher schools. Revista Inclusiones, 8(1), 97-105.

Prikhozhaya, L. I. (2019). Telephone etiquette in the aspect of intercultural communication. Philological aspect, 1, 93-100.

Radbil, T. B. (2014). National models of communication in linguistic coverage: to the formulation of the problem. Communication Research, 1, 41-50.

Seregina, T., Zubanova, S., Druzhinin, V., and Shagivaleeva, G. (2019). The Role of Language in Intercultural Communication. Space and Culture, India, 7(3), 243-253.

Shenberger, I. I. (2008). The causes of misunderstandings at the linguistic level in the process of intercultural communication. Bulletin of Tomsk State University, 309, 24-27.

Sinyachkin, V. P. (2017). Intercultural communication. Study of sign linguistic and non-linguistic communication. Moscow: Peoples' Friendship University of Russia.

Tsymbalyuk, E. I., Romanenko, N. M., Romanenko, A. V., and Volkova, M. A. (2021). Psychological and Pedagogical Conditions Conducive to the Formation of Intercultural Competence Among Students from Different Countries. Journal of Language and Linguistic Studies, 17, 1392-1409.

Vedenskaya, T. E. (2015). The linguistic aspect of the problems of intercultural communication. In Language and text: structure, discourse, translation. Moscow: Moscow Aviation Institute (National Research University).

Vladimirova, T. E. (2012). Intercultural communication: linguistic aspect. Voronezh State University Bulletin. Series: Linguistics and Intercultural Communication, 2, 7-12.

Yi, X. (2020). A study on the cultivation model of intercultural communicative competence in foreign language teaching. In 2020 International Conference on Information Science and Education (ICISE-IE), 9-12. 